\title{
Illumination coherence engineering and quantitative phase imaging
}

\author{
José A. Rodrigo*1 and Tatiana Alieva ${ }^{1}$ \\ ${ }^{1}$ Universidad Complutense de Madrid, Facultad de Ciencias Físicas, Ciudad Universitaria s/n, Madrid 28040, Spain \\ *Corresponding author: jarmar@fis.ucm.es
}

compiled: May 25, 2015

\begin{abstract}
Partially coherent illumination provides significant advantages such as speckle-free imaging and enhanced optical sectioning in optical microscopy. The knowledge of the spatial and temporal coherence is crucial to obtain accurate quantitative phase imaging (QPI) of specimens such as live cells, micron-sized particles, etc. In this Letter, we propose a novel technique for illumination coherence engineering. It is based on a DMD projector providing fast switchable both multi-wavelength and spatial coherence design. Its performance is experimentally demonstrated for QPI with different spatial coherence states.

OCIS codes: (100.3010) Image reconstruction techniques; (030.0030) Coherence and statistical optics; (100.5070) Phase retrieval; (110.0180) Microscopy; (120.4630) Optical inspection

http://dx.doi.org/10.1364/XX.99.099999
\end{abstract}

The fact that coherence properties of light illumination play a crucial role in microscopic image formation and have to be adapted to the observable specimen is well-known. Any modern standard widefield microscope equipped with halogen lamp allows for changing the spatial coherence of the illumination via condenser aperture diaphragm (iris) manipulation and its temporal coherence via a color filter. The earlier development of quantitative imaging, which requires phase recovery of the light beam scattered by the specimen, has forced the use of monochromatic and spatially coherent laser illumination sources [1]. Nevertheless, the image obtained under highly coherent illumination suffers from speckle noise and cross-talk caused by objects located in the same or surrounding depth layers. This important disadvantage waked up the interest to use partially coherent light, see for example [2-4]. The development of successful non-interferometric techniques for phase recovery under partially coherent illumination [5-7], together with fast algorithms for their digital implementation, have made this type of illumination competitive to the conventional coherent light microscopy [1]. Nevertheless, the knowledge of the illumination coherence properties is crucial for accurate image reconstruction of the specimen using these techniques. Moreover, it is preferable that the light coherence characteristics can be changed in a fast and programmable way in order to adapt them to a particular specimen or technique applied for quantitative imaging. It requires a beam shaping tool for control of the spatial and temporal coherence of the illumination beam. For instance, a programmable spatial light modulator (SLM) can be used for this task. Multimedia DLP projectors based on DMD (digital micro-mirror device SLM) technology are a good option because they are fast in the generation of color amplitude images (masks). Several works have demonstrated the use of multimedia projectors for different applications in optical microscopy [8-10]. Note that they can be easily incorporated in optical microscopes and are cost-effective. In [8] two types of multimedia projectors (SLM technologies: Liquid crystal display and digital micro-mirror device) have been proposed for obtaining contrast-enhanced imaging (such as dark-field, Rheinberg optical-staining and oblique illumination microscopy) of transparent samples in a conventional widefield microscope. In such a case the multimedia projector allows for generating of a reconfigurable condenser-lens diaphragm, thus replacing the mechanical iris. While in [9] the projector has been applied to create a light pattern into the sample plane for selective fluorescence microscopy. DMD projectors have also been used for structured illumination to obtain image super-resolution in optical microscopy [10].

In this work we propose the use of DLP projector technology (DMD) for fast and programmable control of temporal and spatial coherence of microscope illumination, thus providing wavelength switchable specklenoise-free imaging. To the best of our knowledge, the use of this technology for illumination coherence engineering in microscopy has not been reported. We experimentally demonstrate that quantitative phase imaging with partially coherent illumination is possible even if the spatial coherence function has no circular symmetry. The amplitude and phase of the object beam are retrieved by measuring several defocused images used as constraint images in the iterative algorithm reported in 
(a) Microscope, illumination and measurement setups:

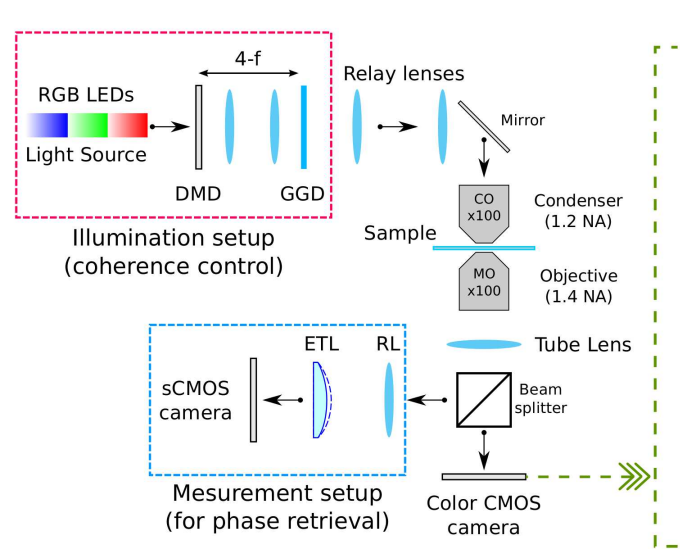

(b)

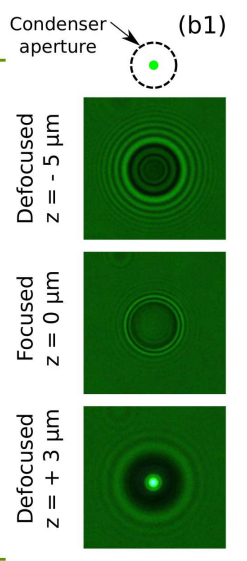

Brightfield images for different light coherence states:

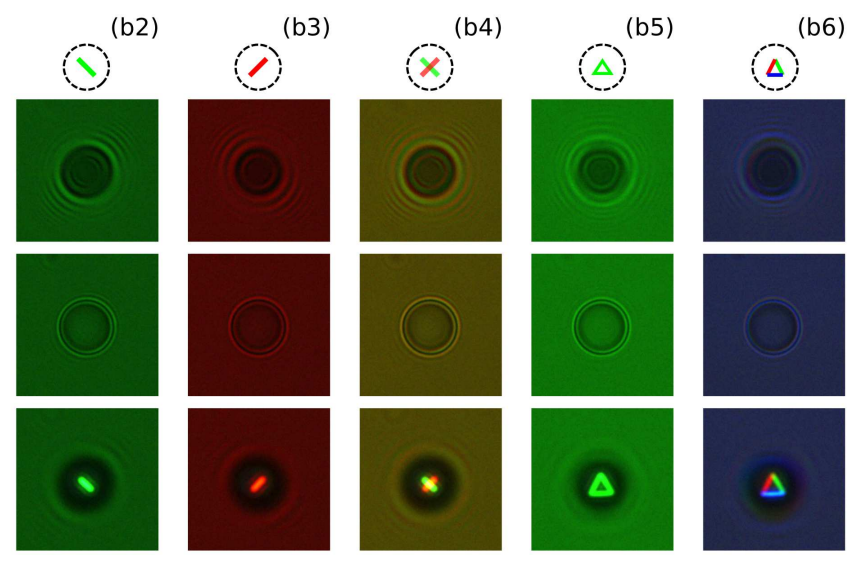

Fig. 1. (Color online) (a) Sketch of the setup for illumination coherence engineering, transmission microscope and phase retrieval system. All the relay lenses and tube lens are achromatic doublets (Thorlabs, N-BK7 glass, focal lengths of $150 \mathrm{~mm}$ ). ETL is a low dispersion varifocal lens. (b) Measured brightfield images of the sample (4.7 $\mu \mathrm{m}$ polystyrene bead) for three focusing distances and six different coherence states as indicated in (b1)-(b6). Media 1 shows the defocused images for each case.

[7], which takes into account the spatial and temporal coherence characteristics of the illumination.

The DLP projector allows for dynamic generation of different images into the back focal plane of the Köhler condenser, as in [8]. We consider a DLP projector (LG PA70G, 750 ANSI lumens of power, Texas Instruments DMD) with three LED sources providing quasimonochromatic RGB (red-green-blue) channels. The experimental setup is sketched in Fig. 1(a), where all optical elements are fixed. In our case, the projection lens of the projector is replaced by a $4-f$ setup $(\times 1 \mathrm{Ke}-$ plerian telescope) that images the DMD into a static ground glass diffuser (GGD, Thorlabs N-BK7 glass, 1500 grit), which warrants for light spatially incoherent. The image at the GGD is relayed $(\times 1$ Keplerian telescope) into the back focal plane of the condenser (Nikon PlanAchromat, 1.25 NA, $\times 100$, Oil). According to the Van Cittert-Zernike theorem [11] the cross-spectral density $J(\mathbf{r}, \nu)$ of the illumination beam (at the sample $x-y$ plane), which describes the correlation of the field at two points separated by a vector $\mathbf{r}=(x, y)$, is related to the spectral density of the projected image $I_{s}(\mathbf{r}, \nu)$ through the Fourier transform

$$
J(\mathbf{r}, \nu) \propto \int I_{s}\left(\mathbf{r}^{\prime}, \nu\right) \exp \left[\mathrm{i} k \mathbf{r} \mathbf{r}^{\prime} / \mathrm{f}\right] \mathrm{d} \mathbf{r}^{\prime} .
$$

Here $k=2 \pi \nu / c=2 \pi n / \lambda_{0}$ is a wavenumber, $\nu$ is temporal frequency, with $\lambda_{0}$ being the wavelength, $n$ is the refractive index of the condenser lens immersion medium (e.g. oil Cargille Type B, $n=1.51$ ), $\mathrm{f}$ is the focal length of the condenser lens. Thus, it is assumed that the sample illumination is uniform and follows the Schell model $[7,12]$. For example, if a centered circle of radius $a$ is projected then the complex degree of coherence (for uniform monochromatic illumination) corresponds to the wellknown Besinc function: $J(\mathbf{r}, \nu)=2 J_{1}(k a r / f) /(k a r / f)$. Other projected images $I_{s}(\mathbf{r}, \nu)$ generate other coherence states $J(\mathbf{r}, \nu)$. Not only the geometric form of the projected image $I_{s}(\mathbf{r}, \nu)$ but also the shape of its intensity and color distributions define the illumination coherence and therefore the image formation. We recall that the temporal coherence is controlled by choosing the appropriate color spectrum of the projected image.

In our experimental study, brightfield focused and defocused images of the sample are acquired by a color camera (Thorlabs-DCC1240C) for monitoring tasks and qualitative analysis, see Fig. 1(a). While for quantitative phase imaging several defocused images are measured by using the setup developed in [7] comprising a fast electrically tunable lens (ETL, Optotune EL-1030-C) and monochrome high-resolution sCMOS camera (Hamamatsu, Orca Flash 4.0, 16-bit gray-level, pixel size of $6.5 \mu \mathrm{m}$ ), see Fig. 1(a). We use a high numerical aperture objective lens (Olympus UPLSAPO, 1.4 NA, 100×, Oil). Fig. 1(b) and Media 1 show the diffraction patterns of the light scattered by a polystyrene sphere $(4.7 \mu \mathrm{m}$ bead diameter, Spherotech Lot. AD01) immersed in distiller water. Specifically, six different illumination states corresponding to $I_{s}(\mathbf{r}, \nu)$ : Green circle (e.g. iris), green and red rectangles tilted an angle $\pm 45^{\circ}$, green triangle and RGB triangle are considered. These images indicated in Fig. 1(b) (top row) are projected into the back focal plane of the condenser. The diffraction patterns reveal the change in the light coherence state due to the color form and shape of the projected images $I_{s}(\mathbf{r}, \nu)$. For instance, as expected, they are symmetric when $I_{s}(\mathbf{r}, \nu)$ is a centered circle [Fig. 1(b1)] and asymmetric in the other cases. In particular, well-defined diffraction fringes are observed only in the direction corresponding to the smallest side of the projected rectangle image [see Fig. 1(b2) and 1(b3)], where the coherence is significantly higher. However, for the best focused sample plane (at $z=0$ the object is in the focal plane of the objective) such a difference in the diffraction pattern is 
not appreciated. This fact supports the assumption of Schell-model illumination beam, according to which the intensity distribution at this imaging plane does not depend of the spatial coherence state of the illumination beam [7].

The choice of color of the projected image $I_{s}(\mathbf{r}, \nu)$ allows for controlling the temporal coherence of the illumination and performing multi-wavelength sample study. Fig. 2(a) displays the measured light spectra of the DLP projector, the central wavelengths are: $\lambda_{0}=622$, 518 and $450 \mathrm{~nm}$ with $\triangle \lambda=16,30$ and $20 \mathrm{~nm}$ being the full width at half maximum (FWHM) of the spectrum, respectively. We recall that in the case of quasimonochromatic LED source $\left(\Delta \lambda \ll \lambda_{0}\right)$ with nearly Gaussian spectrum, the coherence length is often approximated as $l_{c} \approx 0.88 \lambda_{0}^{2} / \Delta \lambda$ [13]. Combining three RGB channels a temporally low coherent illumination is obtained. It has been found beneficial for a recently proposed tomographic technique [4]. On the other hand, by choosing consecutively one of the RGB channels, dispersion analysis of the sample or the surrounding medium can also be performed. In the case of an homogeneous dielectric bead, for example, such an analysis can be done simultaneously by using the design of the illumination spatial coherence state considered in Fig. 1(b4). It is clearly observed that the maxima and minima of the red and green diffraction fringes do not coincide, see top image in Fig. 1(b4). More complex diffraction patterns, displayed in Fig. 1(b5) and 1(b6) (see also Media 1), are obtained for a triangle projected image $I_{s}(\mathbf{r}, \nu)$. We also underline that the spherical bead works as a microlens and therefore the input $I_{s}(\mathbf{r}, \nu)$ is imaged at its focal plane as observed in the third row of Fig. 1(b). This fact might be particularly interesting for micro-pattering in lithography applications.

The later examples illustrate the versatility of the illumination setup for light coherence control. As we have previously mentioned, the knowledge of the illumination coherence properties is important for quantitative phase imaging. In particular, the temporal coherence limits the defocus interval appropriate for application of phase retrieval techniques based on defocussing [7]. While, the knowledge of the function describing the spatial coherence is required for correct reconstruction of the object (sample) wavefield. Let us now study quantitative phase imaging for a fixed wavelength $\lambda_{0}=518 \mathrm{~nm}\left(l_{c} \sim 9 \mu \mathrm{m}\right)$ in the case of three basic spatial coherence states, $I_{s}(\mathbf{r}, \nu)$ with shapes indicated in Fig. 2: Circle 2(b), rectangle $2(\mathrm{c})$ and triangle $2(\mathrm{~d})$. The intensity distribution of the partially coherent diffracted beam at the detector plane [e.g. sCMOS camera in the measurement setup, Fig. 1(a)] for a certain ETL focal length, $\mathrm{f}_{m}$, is given by the expression:

$$
I_{P C}^{m}(\mathbf{r}, \nu) \propto \int I_{C}^{m}\left(\mathbf{r}^{\prime}, \nu\right) I_{s}\left[\left(\mathbf{r}-\mathbf{r}^{\prime}\right) S_{m}, \nu\right] \mathrm{d} \mathbf{r}^{\prime},
$$

see [7] for further details. Here, $S_{m}=\mathrm{f}_{m} /\left(d-\mathrm{f}_{m}\right)$

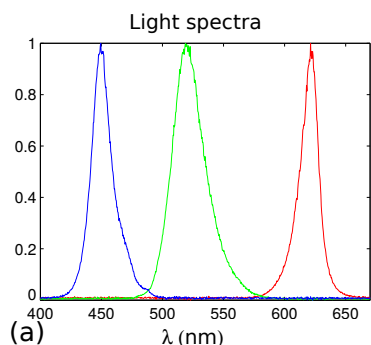

Beam at the input aperture of condenser
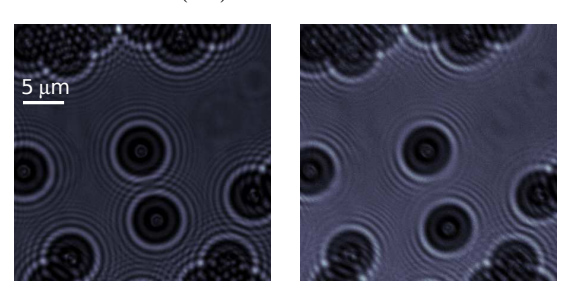

(c)

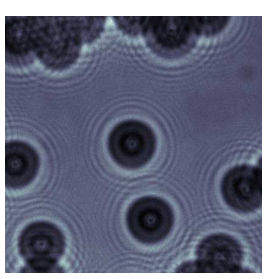

Measured intensity for $(d)$

Fig. 2. (Color online) (a) Measured illumination light spectra (Ocean Optics USB2000+ spectrometer). (b)-(d) Amplitude of the cross-spectral density $J(\mathbf{r}, \nu)$ associated with three images $I_{s}(\mathbf{r}, \nu)$ projected into the back focal plane of the condenser lens for $\lambda_{0}=518 \mathrm{~nm}$. Third row shows some of the measured diffraction patterns (at $\triangle z=6 \mu \mathrm{m}$ ) used for phase retrieval, for each spatial coherence state.

is a scaling factor where $d$ is a fixed distance between the ETL and the sCMOS camera. Specifically, the expression Eq. (2) is the convolution between $I_{s}(\mathbf{r}, \nu)$ and $I_{C}^{m}(\mathbf{r}, \nu)$ that corresponds to the intensity distribution which would be obtained using ideal (speckle-free) spatially coherent illumination. The shift of the imaged plane is given as a function of the focal length of the ETL, the effective magnification of the objective-tube lenses $(M=83)$ and focal length $\mathrm{f}_{R L}$ of the relay lens [see Fig. 1(a)] as it follows: $\triangle z=n_{s} \mathrm{f}_{R L}^{2}\left(\mathrm{f}_{m}-d\right) / \mathrm{f}_{m} d M^{2}$, where $n_{s}=1.33$ is the refractive index of the sample immersion medium (water), see [7]. The range of variation of the focal length $\mathrm{f}_{m}$ depends on temporal and spatial coherence properties of the source. The measurement is performed inside the coherence gate, thus the defocussing range is mostly limited by $\left|\triangle z_{\max }\right|<l_{c}$. Note that as the coherence becomes low the defocussing range in the measurement has to be shorter. In this range the object's diffraction patterns have to be well resolved and sufficiently vary with defocus such that the iterative algorithm can recover the sample phase information. Fig. 2 displays three of the diffraction patterns (at $\triangle z=6 \mu \mathrm{m})$ collected by the sCMOS camera for the considered spatial coherence states indicated in Fig. 2(b)$2(\mathrm{~d})$. The change in the symmetry of the diffraction pattern for each case is clearly distinguished.

To retrieve the phase distribution of the object beam, nine diffraction patterns $I_{P C}^{m}(\mathbf{r}, \nu)(m=1, \ldots, 9)$ were measured for each case. These diffraction patterns are used as constraint images in the iterative algorithm reported in [7], which is similar to Gerchberg-Saxton one but running for the Fresnel transform (free-space propagation) with an additional deconvolution routine to take 


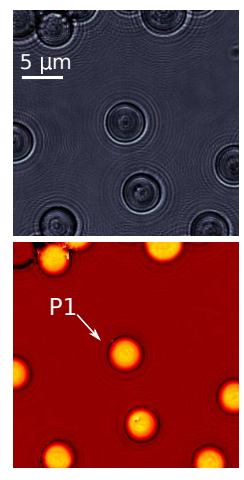

(a)

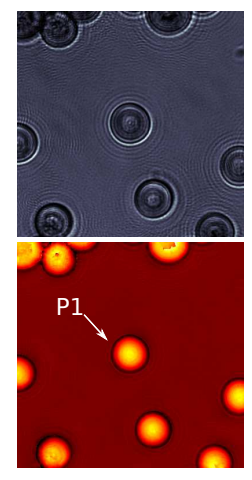

(b)

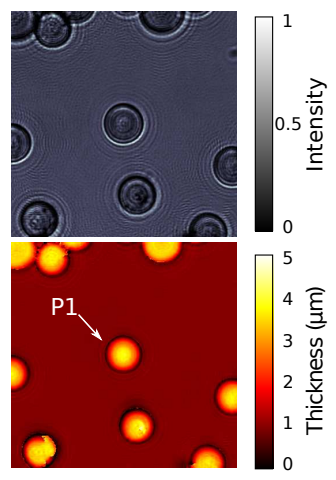

(c)

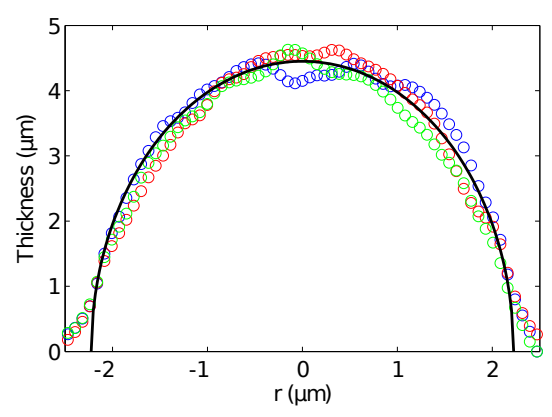

$\lambda_{0}=518 \mathrm{~nm}$

Thickness profile for P1 with:

- $J(r, v)$ in (a)

- $J(r, v)$ in (b)

- $J(\mathbf{r}, v)$ in (c)

(d)

Fig. 3. (Color online) (a)-(c) Reconstructed intensity image and thickness distributions of the polystyrene beads. (d) Thickness profiles of the bead P1 fit well to the expected one (continuous line) for each coherence state $J(\mathbf{r}, \nu)$ in the cases: Circle, rectangle and triangle $I_{s}(\mathbf{r}, \nu)$.

into account the effects of the spatial coherence state associated to $I_{s}(\mathbf{r}, \nu)$. Indeed, the algorithm retrieves both the intensity and phase of the object wavefield thus allowing for numerical refocusing if needed. Here we use the same procedure as reported in [7] (see Section 2) and images $I_{P C}^{m}(\mathbf{r}, \nu)$ of $1024 \times 1024$ pixels, the convergence is typically reached after 10 iterations in a few seconds $(20 \mathrm{~s})$. From the phase $\phi(\mathbf{r})$ of the retrieved object wavefield, the particle thickness $t(\mathbf{r})$ is determined as it follows: $t(\mathbf{r})=\phi(\mathbf{r}) \lambda_{0} / 2 \pi \triangle n$, where $\triangle n=n_{o}-n_{s}$ is the refractive index difference between the bead $\left(n_{o}=1.60\right.$ at $\lambda_{0}=518 \mathrm{~nm}$ ) and its surrounding medium (water, $n_{s}=1.33$ ) [1]. For accurate phase unwrapping we applied the algorithm reported in [14]. The retrieved intensities at the best focused image plane and the corresponding reconstructed thickness distributions for each spatial coherence state of the illumination are shown in Fig. 3. These results are in good agreement with the expected thickness profile of a spherical bead, as displayed in the third row of Fig. 3. Because the particle is spherical in shape one can estimate both the radius $(R)$ of the bead and $\triangle n$ by linear fitting of the plot corresponding to the square of the phase

$$
\phi^{2}(x, y)=\frac{(4 \pi \triangle n)^{2}}{\lambda_{0}^{2}}\left(R^{2}-r^{2}\right),
$$

against $r^{2}=x^{2}+y^{2}$. Note that this procedure is of- ten applied for testing in quantitative phase imaging, see for example $[6,7,15]$. The diameter of the bead (P1 in Fig. 3) is about $4.4 \pm 0.2 \mu \mathrm{m}$ for all considered illumination cases.

The latter experimental results demonstrate that quantitative phase imaging (QPI) is possible even using partially coherent illumination whose spatial coherence $J(\mathbf{r}, \nu)$ exhibits rather different symmetry with respect the conventional circular one, Fig. 2(b)-2(d). While here we have consider the QPI using defocussing-based iterative phase retrieval technique the knowledge of the temporal and spatial coherence of the illumination is also required for the application of other retrieval techniques such as TIE [5] and interferometric approaches [3, 4]. The use of tunable light coherence brings promising advantages for QPI yet to study and exploit. A part from QPI, the fast programmable control of the illumination coherence properties via DMD-based LED-illumination projection technology provides important benefits for multi-functional microscopy involving sample monitoring or inspection, and photo-video-micrography, etc.

Spanish Ministerio de Economía y Competitividad is acknowledged for funding the project TEC2011-23629.

\section{References}

[1] P. Ferraro, A. Wax, and Z. Zalevsky, eds., Coherent Light Microscopy: Imaging and Quantitative Phase Analysis (Springer, 2011).

[2] B. Redding, M. A.Choma, and H. Cao, Nat Photon 6, 355-359 (2012).

[3] B. Kemper, S. Sturwald, C. Remmersmann, P. Langehanenberg, and G. von Bally, Opt. Lasers Eng. 46, 499 - 507 (2008).

[4] T. Kim, R. Zhou, M. Mir, S. D. Babacan, P. S. Carney, L. L. Goddard, and G. Popescu, Nat Photon pp. 256263 (2014).

[5] D. Paganin and K. A. Nugent, Phys. Rev. Lett. 80, 2586-2589 (1998).

[6] K. G. Phillips, C. R. Velasco, J. Li, A. Kolatkar, M. Luttgen, K. Bethel, B. Duggan, P. Kuhn, and O. McCarty, Frontiers in Oncology 2 (2012).

[7] J. A. Rodrigo and T. Alieva, Opt. Express 22, 13472 13483 (2014).

[8] E. C. Samson and C. M. Blanca, New Journal of Physics 9, 363 (2007).

[9] J. N. Stirman, M. M. Crane, S. J. Husson, A. Gottschalk, and H. Lu, Nat. Protocols 7, 207-220 (2012).

[10] D. Dan, M. Lei, B. Yao, W. Wang, M. Winterhalder, A. Zumbusch, Y. Qi, L. Xia, S. Yan, Y. Yang, P. Gao, T. Ye, and W. Zhao, Sci. Rep. 3 (2013).

[11] M. Born and E. Wolf, Principles of Optics (Cambridge University Press, UK, 2006).

[12] A. C. Schell, "The multiple plate antenna," Ph.D. thesis, Massachusetts Institute of Technology (1961).

[13] J. W. Goodman, Statistical Optics (Wiley\&Sons, NY, 2000).

[14] M. A. Herráez, D. R. Burton, M. J. Lalor, and M. A. Gdeisat, Appl. Opt. 41, 7437-7444 (2002).

[15] T. J. McIntyre, C. Maurer, S. Fassl, S. Khan, S. Bernet, and M. Ritsch-Marte, Opt. Express 18, 14063-14078 (2010). 


\section{References}

[1] P. Ferraro, A. Wax, and Z. Zalevsky, eds., Coherent Light Microscopy: Imaging and Quantitative Phase Analysis (Springer, 2011).

[2] B. Redding, M. A.Choma, and H. Cao, "Speckle-free laser imaging using random laser illumination," Nat Photon 6, 355-359 (2012).

[3] B. Kemper, S. Sturwald, C. Remmersmann, P. Langehanenberg, and G. von Bally, "Characterisation of light emitting diodes (LEDs) for application in digital holographic microscopy for inspection of micro and nanostructured surfaces," Opt. Lasers Eng. 46, 499 - 507 (2008).

[4] T. Kim, R. Zhou, M. Mir, S. D. Babacan, P. S. Carney, L. L. Goddard, and G. Popescu, "White-light diffraction tomography of unlabelled live cells," Nat Photon pp. 256-263 (2014).

[5] D. Paganin and K. A. Nugent, "Noninterferometric phase imaging with partially coherent light," Phys. Rev. Lett. 80, 2586-2589 (1998).

[6] K. G. Phillips, C. R. Velasco, J. Li, A. Kolatkar, M. Luttgen, K. Bethel, B. Duggan, P. Kuhn, and O. McCarty, "Optical quantification of cellular mass, volume and density of circulating tumor cells identified in an ovarian cancer patient," Frontiers in Oncology 2 (2012).

[7] J. A. Rodrigo and T. Alieva, "Rapid quantitative phase imaging for partially coherent light microscopy," Opt.
Express 22, 13472-13483 (2014).

[8] E. C. Samson and C. M. Blanca, "Dynamic contrast enhancement in widefield microscopy using projectorgenerated illumination patterns," New Journal of Physics 9, 363 (2007).

[9] J. N. Stirman, M. M. Crane, S. J. Husson, A. Gottschalk, and $\mathrm{H}$. Lu, "A multispectral optical illumination system with precise spatiotemporal control for the manipulation of optogenetic reagents," Nat. Protocols 7, 207-220 (2012).

[10] D. Dan, M. Lei, B. Yao, W. Wang, M. Winterhalder, A. Zumbusch, Y. Qi, L. Xia, S. Yan, Y. Yang, P. Gao, T. Ye, and W. Zhao, "DMD-based LED-illumination Super-resolution and optical sectioning microscopy," Sci. Rep. 3 (2013).

[11] M. Born and E. Wolf, Principles of Optics (Cambridge University Press, UK, 2006).

[12] A. C. Schell, "The multiple plate antenna," Ph.D. thesis, Massachusetts Institute of Technology (1961).

[13] J. W. Goodman, Statistical Optics (Wiley\&Sons, NY, 2000).

[14] M. A. Herráez, D. R. Burton, M. J. Lalor, and M. A. Gdeisat, "Fast two-dimensional phase-unwrapping algorithm based on sorting by reliability following a noncontinuous path," Appl. Opt. 41, 7437-7444 (2002).

[15] T. J. McIntyre, C. Maurer, S. Fassl, S. Khan, S. Bernet, and M. Ritsch-Marte, "Quantitative SLM-based differential interference contrast imaging," Opt. Express 18, 14063-14078 (2010). 